\title{
NIPPV Effective for Pulmonary Edema
}

CLINICAL QUESTION: Is noninvasive positive pressure ventilation effective in managing patients with acute cardiogenic pulmonary edema?

- BOTTOM LINE: Patients with acute cardiogenic pulmonary edema treated with noninvasive positive pressure ventilation (NIPPV) are less likely than those receiving standard care to die in the hospital or to require mechanical ventilation. $(\mathbf{L O E}=\mathbf{1 a})$

REFERENCE: Peter JV, Moran JL, Phillips-Hughes J, Graham P, Bersten AD. Effect of non-invasive positive pressure ventilation (NIPPV) on mortality in patients with acute cardiogenic pulmonary oedema: a metaanalysis. Lancet 2006;367:1155-1163.

n STUDY DESIGN: Meta-analysis (randomized controlled trials)

- SETTING: Inpatient (any location)

- SYNOPSIS: This team systematically reviewed multiple databases, using a sensible search strategy,q to find 23 small randomized controlled trials of NIPPV. NIPPV included continuous positive airway pressure or bilevel ventilation. The data were extracted independently by 2 investigators, with discrepancies resolved by consensus. The authors don't report if the decision to include or exclude studies was similarly done independently. Additionally, they don't say if they looked for unpublished studies. The eligible studies included more than 1300 patients with cardiogenic pulmonary edema. NIPPV was more effective than standard care in preventing in-hospital mortality $(11.7 \%$ vs $21.3 \%$; number needed to treat $[\mathrm{NNT}]=11$; 95\% CI, 7 - 21), with no difference between continuous positive airway pressure and bilevel ventilation. Similarly, patients receiving NIPPV required mechanical ventilation less frequently ( $11.9 \%$ vs $28.1 \%$; NNT $=7$; 5 - 9), with no difference in outcomes between continuous positive airway pressure and bilevel ventilation. The data were fairly consistent across the studies. Since there is a possibility of publication bias in favor of positive results, the results of a mega-trial (if one occurs) may not look this good. 